\title{
Thermal analysis of high power LED light
}

\author{
Ma Yunpeng ${ }^{1}$, Zhou Quan ${ }^{2}$ \\ ${ }^{1}$ School of Aeronautic Science and Engineering, Beihang University, Beijing, 100191, China \\ ${ }^{2}$ School of Aeronautic Science and Engineering, Beihang University, Beijing, 100191, China \\ amyp@buaa.edu.cn, ${ }^{b}$ zhouquan@buaa.edu.cn
}

Keywords: high-power LED; heat dissipation; simulation analysis

\begin{abstract}
As a typical green lighting source, but most of high-power LED input power is converted into heat, heat dissipation has important influence on its performance. For the LED lamp radiator, the heat conduction mode is obtained. By the finite element simulation analysis and thermal analysis of natural convection radiator, the steady-state temperature distribution of the radiator is simulated, and compared the simulation analysis and thermal test results. Results show that the design of the radiator satisfies the requirement of heat dissipation, and verifies the correctness of thermal resistance analysis method.
\end{abstract}

\section{Introduction}

Compared with the traditional incandescent lamp and fluorescent lamp, high power white LED has high brightness, low power consumption, long life, good directivity, fast response, no radiation, green as a new generation of green environmental protection solid lighting source.

LED produces light by electronic transition between different energy band, and the light does not contain the infrared part, and its heat cannot disappear by radiation. At present the LED luminous efficiency can reach $10 \%-20 \%$, the rest of energy is converted into heat. Therefore, used multiple single LED chip assembly into a module, a high power LED temperature will rise rapidly, which leads to working voltage decrease, light intensity decrease, longer wavelengths of light, speed droop. So, the research on high power LED heat dissipation is of important theoretical and engineering value. Now the commonly thermal radiation are: air cooling, heat pipe cooling and thermoelectric refrigeration, etc. By using the theory of evaporation heat absorption refrigeration, heat pipe has good cooling effect.

By the finite element simulation analysis and thermal analysis of natural convection radiator, the steady-state temperature distribution of the radiator is simulated, and compared the simulation analysis and thermal test results. Results show that the design of the radiator satisfies the requirement of heat dissipation, and verifies the correctness of thermal resistance analysis method.

\section{The thermal calculation of LED lighting lamps and lanterns}

The structure of a 50w white LED. A 50W high-power white light LED lighting is mainly composed of radiator, LED chip, the condenser cover, lamp holder and the power supply, chimney.

As shown in Figure 2、 Figure 3, a radiator is designed with simple structure, low cost, easy production. The radiator use aluminum fins which has good thermal conductivity performance, and the thermal conductivity is $150 \mathrm{~W} / \mathrm{m}^{2} \cdot k$ ), metal circuit board and radiator base with high thermal conductivity silicone adhesive coefficient which thermal conductivity is $3.8 \mathrm{~W} / \mathrm{m}^{2} \cdot k$ ). 


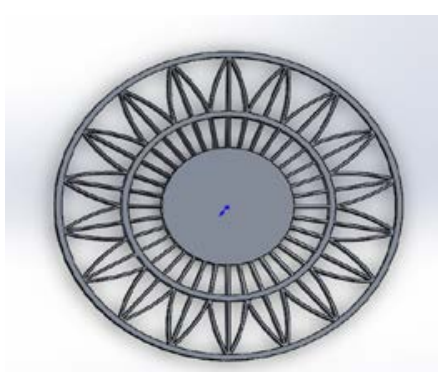

Fig 1 the structure of radiator

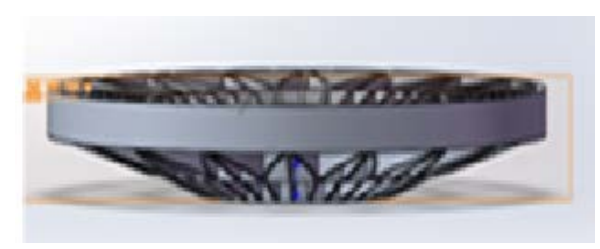

Fig 2 the side view of radiator

Thermal analysis model. The 50W high power LED is a more chip components, composed of five $10 \mathrm{~W}$ chip packages. Calculation is simplified to: all chip in series connection, and array in the same circuit boards, chip specifications are exactly the same, work temperature and consumed power completely consistent, ignore the thermal interaction between the chip, so similar to parallel circuit chip thermal resistance model of type. As shown in Fig $3 R_{J C}$ is the thermal resistance between the chip and internal heat sink, $R_{J C}$ is the thermal resistance between internal heat sink thermal resistance metal circuit board, $R_{B A}$ is the thermal resistance between circuit board and radiator.

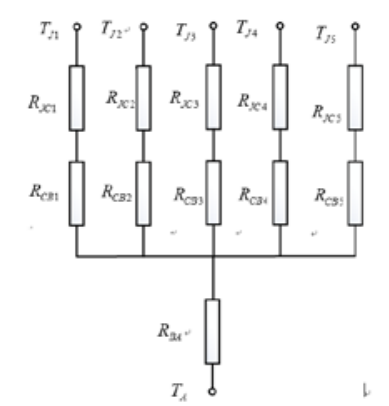

Figure 3 multichip LED equivalent thermal model

We can see that the multichip LED integrated encapsulation of the equivalent heat path is than each LED chip thermal resistance connect in parallel and then connection with thermal resistance, thermal resistance and convection radiator thermal resistance in series.

The parallel formula:

$$
\frac{1}{R_{J C}}=\frac{1}{R_{J C 1}}+\frac{1}{R_{J C 2}}+\frac{1}{R_{J C 3}}+\frac{1}{R_{J C 4}}+\frac{1}{R_{J C 5}}
$$

Because each chip is the same, so:

$$
R_{J C}=\frac{R_{J C 1}}{5}
$$

And similarly:

$$
R_{C B}=\frac{R_{C B 1}}{5}
$$

The total thermal resistance of the model:

$$
R_{J A}=\frac{R_{J C 1}}{5}+\frac{R_{C B 1}}{5}+R_{B A}
$$

According to the concept of thermal resistance, the LED junction temperature can be obtained:

$T_{J}=T_{A}+R_{J A} \times P_{D}$

$T_{A}$ is the environmental temperature, $P_{D}$ is the chip's total power consumption. 
$R_{J B}=8^{\circ} \mathrm{C} / \mathrm{W}, T_{A}=25^{\circ} \mathrm{C}$,

Thermal resistance of thermal conductive adhesive $\mathrm{R} 1=1.8^{\circ} \mathrm{C} / \mathrm{W}$.

$T_{J} \leq 65^{\circ} \mathrm{C}$, We can get

$R_{S R} \leq \frac{65-25}{5}-2-1.8=6.2^{\circ} \mathrm{C} / \mathrm{W}$

Radiator thermal resistance $R=5.5^{\circ} \mathrm{C} / \mathrm{W}$;

We can get the available minimum heat dissipation area of the radiator

$$
S=\frac{1}{R h}=0.302 m^{2}
$$

\section{The heat flow field analysis of LED lamps and lanterns the cooling structure}

This article uses the finite element method to simulate the steady-state heat flow field distribution of chip

Radiator steady state thermal analysis. The steady state thermal analysis of LED lamps and lanterns. The temperature distribution should meet with heat source of heat conduction differential equation when LED chip works in a state performance.

$$
\frac{\partial^{2} T}{\partial x^{2}}+\frac{\partial^{2} T}{\partial t^{2}}+\frac{\partial^{2} T}{\partial z^{2}}+\frac{q}{k}=0
$$

$\mathrm{T}$ is the temperature, $\mathrm{q}$ is the heat per unit volume, $\mathrm{k}$ is the coefficient of thermal conductivity.

Using finite element software simulation, we define that the environment temperature $25{ }^{\circ} \mathrm{C}$, radiator's convective heat transfer coefficient $5 \mathrm{~W} / \mathrm{m}^{2} \cdot k$, the chip P-N junction is the heat source, Heat generation rate for unit volume $2.04 e 8 \mathrm{~W} / \mathrm{m}^{3}$. From the Fig 4 , we can find the highest temperature area concentrated in the center of chip, and the highest temperature is $62.3^{\circ} \mathrm{C}$.

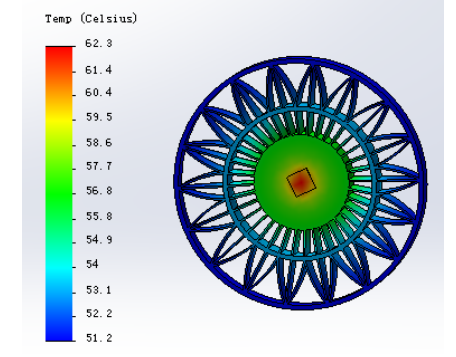

Fig 4 Steady-state temperature field distribution of the radiator

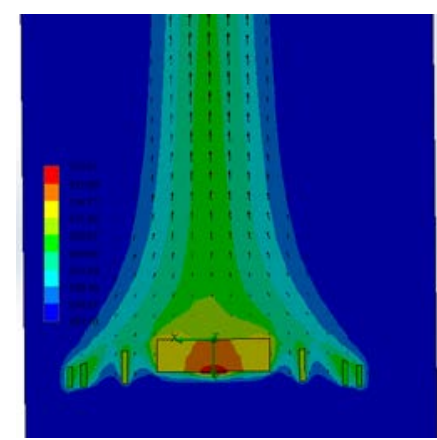

Fig 5 Temperature distribution of the radiator heat flow simulation

\section{Conclusion}

On the basis of the establishment of high-power LED thermal resistance model, this paper calculated the minimum effective cooling area. Through the simulation and experiment, the aluminum finned radiator design is verified to fulfill the requirements of the engineering application under the $25{ }^{\circ}$ Cenvironment temperature within full working capacity, and the chip maximum junction can be controlled within $65{ }^{\circ} \mathrm{C}$. 


\section{References}

[1] Curran J A ,Clyn T W et al . The Thermal Conductivity of Plasma Electrolytic Oxide Coatings on aluminum and Magnesium [J].Surface and Coatings Technology, 2005.

[2] Arik M ,Weaver S. Chip Scale Thermal Management of High Brightness LED Packages[J ] . Proc of SPIE, 2004

[3] Zhang Guangchen, Feng Shiw ei, Guo Chunsheng, etal. Thermal analysis of high power LED array system[C]. Pro c. 26th IEEE Semi Therm Symposium, Sant a Clara, CA, 2010

[4] Krames Mr, Shchekin, Oleg B, Mueller Mach, Regina et al. Status and Future of MHigh power Light Emitting Diodes for Solid State Lighting [J] . Journal of Display Technology, June 2007,

[5] Lan Kim, Jong Hwa Choi,Sun HoJangetal Thermal analysis of LED array system with heat pipe[J].Thermochimica Acta,2007

[6] Liu C K,Dai M J,Yu C K,et al.High efficiency silicon-based high power LED package integrated with micro-ther-moelectric device[C].Proc.of IMPACT,2007

[7] S. Moghaddama, M. Radaa, A. Shooshtaria, et al. Evaluation of analytical models for thermal analysis and design of electronic packages. Microelectronics Journal, 2003 